\title{
Predictive validity of the Trauma screening Questionnaire in detecting post-traumatic stress disorder in patients with psychotic disorders
}

Paul A. J. M. de Bont, David P. G. van den Berg, Berber M. van der Vleugel, Carlijn de Roos, Ad de Jongh, Mark van der Gaag and Agnes van Minnen

\section{Background}

Post-traumatic stress disorder (PTSD) is highly prevalent in patients with a psychotic disorder. Because a PTSD diagnosis is often missed in patients with psychosis in routine care, a valid screening instrument could be helpful.

\section{Aims \\ To determine the validity of the Trauma Screening Questionnaire (TSQ) as a screening tool for PTSD among individuals with psychotic disorders.}

\section{Method}

Among 2608 patients with a psychotic disorder, the rate of trauma exposure was determined and the TSQ was administered to screen for PTSD. PTSD status was verified in 455 patients using the Clinician-Administered PTSD Scale (trial registration: ISRCTN 79584912).

\section{Results}

Trauma exposure was reported by $78.2 \%$ of the 2608 patients. PTSD prevalence was estimated at $16 \%(95 \% \mathrm{Cl}$ 14.6-17.4\%) compared with $0.5 \%$ reported in the patients' clinical charts. A TSQ cut-off score of six predicted PTSD with $78.8 \%$ sensitivity, $75.6 \%$ specificity, $44.5 \%$ correct positives and $93.6 \%$ correct negatives

\section{Conclusions}

The TSQ seems to be a valid screening tool for PTSD in patients with a psychotic disorder.

\section{Declaration of interest}

None.

\section{Copyright and usage}

(c) The Royal College of Psychiatrists 2015.
Although the prevalence of trauma exposure and PTSD is high in patients diagnosed with psychotic disorders $(12.4 \%, 95 \% \mathrm{CI}$ $4.0-20.8 \%),{ }^{1}$ there is evidence that in routine care both trauma exposure and the diagnosis of PTSD are often under-reported. ${ }^{2}$ Moreover, in both clinical practice and research, patients with a psychotic disorder are often denied PTSD treatment. ${ }^{3,4}$ The main goal of the present study was to determine whether the Trauma Screening Questionnaire (TSQ) ${ }^{5}$ is a valid screening tool for individuals diagnosed with psychotic disorders to preselect those that might have comorbid PTSD. This was explored in a large sample of this patient population by first screening the patients for DSM-IV PTSD criterion-A trauma exposure. ${ }^{6}$ Then, patients reporting positively on trauma exposure were screened for TSQ scores. The PTSD prevalence in the sample screened was then estimated using the gold standard, the Clinician-Administered PTSD Scale (CAPS), ${ }^{7}$ to assess patients' status on PTSD; for comparative purposes, the PTSD diagnosis recorded in the clinical charts of the participants was checked. The final step was to determine the psychometric properties of the TSQ to predict PTSD in a sample of patients with psychotic disorders.

\section{Method}

The present study was part of the randomised clinical trial Treating Trauma in Psychosis (T.TIP); details of the T.TIP design are published elsewhere ${ }^{8}$ (trial registration: ISRCTN 79584912). In 13 long-term mental healthcare services in the Netherlands, patients with a chart diagnosis of a psychotic disorder or a mood disorder with psychotic features were screened with the TSQ. Patients with elevated scores of PTSD symptoms (TSQ $\geqslant 6$ ) and a randomly selected group of low scorers (TSQ $<6$ ) were subsequently assessed for a diagnosis of PTSD and psychotic disorder. The prevalence of PTSD in the target population was estimated using logistic regression. The validity of the TSQ to predict PTSD was assessed with receiver operating characteristic (ROC) analysis.

Inclusion criteria for screening were: adult patients (aged 18-65 years) in secondary or tertiary mental healthcare with a chart diagnosis of a psychotic disorder or a mood disorder with psychotic features, based on the DSM-IV-TR. ${ }^{6}$ Patients were excluded from the screening if they had insufficient competence in the Dutch language, if their estimated IQ was $\leqslant 70$ (intellectual disability), if they were unable to travel to and from the assessment location and/or if they were in a closed ward or in seclusion. At the end of the screening participants were asked for their consent to contact them again for further interviewing. All inclusion and exclusion criteria applied for the screening procedure remained effective for the interviews.

\section{Measures}

TSQ

We used the TSQ, a 10-item symptom screening tool ${ }^{5}$ derived from the 17-item PTSD Symptom Scale, Self-Report version (PSS-SR). ${ }^{9}$ The TSQ items are answered by ticking 'yes' (symptom is present two times a week or more) or 'no' (symptom is not present or present less than twice a week); the minimum score is zero and the maximum score is 10 . The TSQ has been proven to have good to excellent sensitivity and specificity in assessing potential PTSD in British samples of crime victims and rail crash victims,${ }^{5}$ victims of the 2005 London bombings ${ }^{10}$ and assault victims in Wales. ${ }^{11}$ In these latter studies, a cut-off score of 6 on the TSQ was found to be optimal. The TSQ was translated into Dutch and tested against the CAPS in a Dutch sample of crime and accident victims. ${ }^{12}$ The reliability was good (alpha $(\alpha)=0.85)$. The optimum TSQ cut-off score was found to be 7 , which demonstrated a sensitivity of $87 \%$, a specificity of $69 \%$, a 
positive predictive value (PPV) of $66 \%$, a negative predictive value (NPV) of $89 \%$ and an overall accuracy of $76 \%$. These data suggest that the Dutch version of the TSQ is an effective instrument to screen for PTSD. The TSQ has not yet been tested in individuals with a psychotic disorder.

\section{MINI}

We used the sections for psychotic disorders and mood disorders of the Mini-International Neuropsychiatric Interview-Plus (MINI-Plus) $^{13-15}$ to assess lifetime psychotic disorder. Compared with the Composite International Diagnostic Interview (CIDI), ${ }^{13}$ the MINI shows satisfactory diagnostic properties: (a) a reliability of 0.68 , sensitivity of 0.90 and a specificity of 0.91 in generating a diagnosis of current psychotic syndromes; (b) 0.73, 0.94 and 0.79, respectively, for the diagnosis of major depressive disorder; and (c) $0.65,0.86$ and 0.96 , respectively, for the diagnosis of a current manic episode. The test-retest reliability for psychosis was 0.90 and it was 0.80 for major depressive episode. The interrater reliability for the MINI sections was high: $0.88-1.00$. A validation study ${ }^{14}$ against the Structured Clinical Interview for DSM-IV (SCID-Patient version) supported these results. Thus, the psychometric characteristics of the MINI-Plus make it an appropriate choice for research purposes. Preliminary studies in the Netherlands have demonstrated its suitability for diagnosing psychiatric patients in routine clinical practice. $^{15}$

\section{CAPS}

In the present study, the CAPS categorical diagnosis functions as the test of the accuracy of the TSQ. The 2013 guidelines of the International Society for Traumatic Stress Studies ${ }^{16}$ consider the CAPS to be the gold standard for diagnosing PTSD based on the DSM-IV-TR. A review of the literature on the psychometric properties of the CAPS ${ }^{17}$ indicates that it has excellent reliability $(>0.90)$ and consistency across items, raters and testing occasions. The CAPS also has excellent $(>0.90)$ convergent and discriminant validity, diagnostic utility and sensitivity to clinical change.

\section{Procedures}

Participants were recruited in 2011 and 2012 by teams working in mental health organisations in the Netherlands. The local medical ethics committee approved the study (NL36649.029.12). It was explicitly agreed that not only individuals suspected of having PTSD would be screened with the TSQ but all patients with psychotic disorders. Unlike in previous TSQ studies, ${ }^{5,10,11}$ in the present study the occurrence and/or nature of the participants' traumas was unknown beforehand. That is why for the purpose of the present study we had to administer introductory questions about trauma exposure before administering the TSQ; only patients who reported that they had been exposed to trauma were allowed to fill out the TSQ.

Following our study protocol, ${ }^{8}$ participants first answered the question whether they had 'experienced or witnessed' any PTSD A-criterion event, i.e. ' . . . events that were really frightening, life-threatening, overwhelming or shocking' (see Appendix, Question 1). If they indicated 'no', the screening ended there for that participant. If the answer was 'yes' then they could move on to the next questions. First, they could indicate which (one or more) of six categorised descriptions of possible events fitted their traumatic experiences (Appendix, Question 2): 'sexual activities against your will', 'physical abuse', 'emotional or psychological abuse', 'severe neglect', 'accident/disaster/war' and/or the supplementary category of experiencing 'an episode of psychosis. Together, these six categories constitute a broad conceptualisation of events that might be considered traumatic according to the DSM-IV PTSD criterion A. The categories of sexual abuse, physical abuse and accident/disaster/war represent this conceptualisation beyond any doubt. However, which other events might be considered to meet the DSM-IV criterion A is still under debate. ${ }^{18}$ The screening tool used in the present study also includes the possibility that severe psychological/emotional abuse (for example self or loved ones being threatened with death) or severe neglect (such as insufficient shelter, clothes, food, drink or medical care) and traumatic psychosis and their aftermaths ${ }^{19}$ might be compatible with the PTSD criterion A. The category traumatic psychosis is typical for this patient population, because patients may experience traumatic events because of the content of their psychosis (such as the delusion of being threatened with poisoning) and/or as a result of factors associated with (forced) interventions, such as being held at a police station with violence. In Question 2 patients also indicated, per trauma category, how often they had experienced that particular trauma category: no trauma, a single trauma or multiple traumas.

After the introductory Question 1 and Question 2, the screening moves on to the 10-item TSQ for associated PTSD symptoms (Appendix, Question 3). Note that in the TSQ (Appendix, Question 3) patients are not instructed to indicate for which traumatic experience(s) reported in Question 2 they are filling out the TSQ. The reason for this is that, in this particular patient population, many individuals have experienced multiple traumas and it seems difficult for them to relate their PTSD symptoms to one specific traumatic event. ${ }^{2}$ Therefore, the scores on the TSQ represent PTSD symptom severity associated with the whole range of trauma types and trauma frequencies found in our sample of multi-traumatised, chronically ill out-patients with psychotic disorders.

Patients filled out the questionnaire in the presence of their mental health worker. To complete the process the caregiver filled out a short list of demographic questions, asking about the consenting participant's gender, age, legal status and housing type. Also, mental health services provided a copy of the complete DSM-IV-TR (Axis I to $\mathrm{V}$ with all diagnostic classifications) recorded in the patient's chart. Subsequently, informed and consenting participants with a TSQ cut-off score $\geqslant 6$ and a random selection (SPSS command 'select random sample') of participants with a TSQ score $<6$ entered diagnostic interviewing. These participants were interviewed for verification of the chartregistered lifetime history of a psychotic disorder or mood disorder with psychotic features (MINI-Plus) and of the presence or absence of PTSD (CAPS), both according to the DSM-IV-TR criteria. The CAPS interview was not based on the trauma categories that the patient had reported in screening Question 2 (Appendix). Instead, the CAPS interview was carried out following the CAPS instruction manual. The patient received the CAPS Traumatic Event screening. They selected a maximum of three adverse events that were most closely related to their PTSD symptoms. Then, they described the events in more detail to the interviewer to test whether the event(s) matched PTSD criterion A (CAPS Questions A1 and A2). Only if at least one event fulfilled the PTSD criterion A were the CAPS questions about symptoms (CAPS sections B, C and D), duration (section E) and impairment (section F) administered with respect to the A-criterion trauma(s). The advice of the International Society for Traumatic Stress Studies ${ }^{16}$ was followed concerning symptom score rules and assessing PTSD diagnostic status: ${ }^{20}$ i.e. symptom items with a current frequency score of one or more, and an intensity score of two or more, counted as PTSD symptoms. To fulfil the diagnosis of a current PTSD on the CAPS, a participant had to score one or more symptoms of reliving (B-criterion symptoms), three or more symptoms of avoidance (C-criterion symptoms) 
and two or more symptoms of hyperarousal (D-criterion symptoms) for a minimum duration of 1 month (E) with significant disturbance of functioning as a consequence (F). Figure 1 shows the flow of participants for screening and interviews used to examine the predictive validity of the TSQ in patients with a psychotic disorder.

\section{Data analysis}

To construct ROC curves and estimate the prevalence of PTSD we imputed the CAPS diagnostic outcome (PTSD diagnoses 'yes' or 'no') in the total screened sample $(n=2608)$, using a predictor model. Backward stepwise logistic regression was conducted on the CAPS interviewed sample data $(n=455)$ to select strong and independent predictors of PTSD. Then, we predicted PTSD status per individual in the total screened sample based on the predictor model, using a multiple imputations procedure (by default, five imputations). The estimated PTSD status and the TSQ total scores of all individuals in the sample $(n=2608)$ were then used in ROC analysis to determine the predictive validity of the TSQ. For this, sensitivity, specificity, positive/negative predictive values and optimal cut-off score were calculated. Analyses were conducted using SPSS version 20 .

\section{Results}

\section{Demographic characteristics}

Table 1 presents the characteristics of the participants who were screened $(n=2608)$ and of the subsample who were interviewed

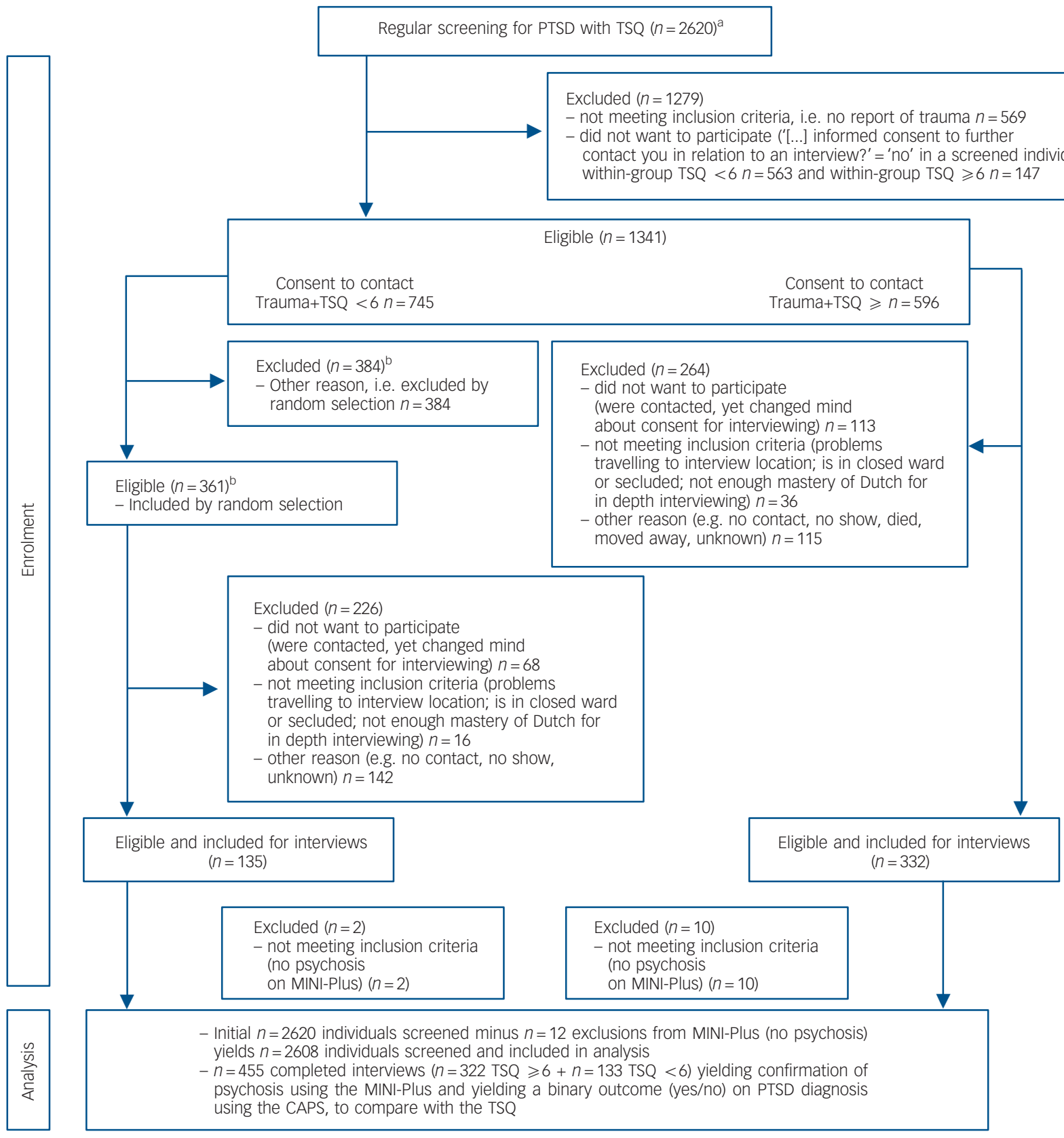

Fig. 1 Flow diagram of the inclusion of participants (screened and interviewed) to examine the predictive validity of the Trauma Screening Questionnaire (TSQ) to detect post-traumatic stress disorder (PTSD) in patients with psychotic disorders.

CAPS, Clinician-Administered PTSD Scale; MINI-Plus, MINI-International Neuropsychiatric Interview-Plus.

a. $n=2620$ screened participants were recruited on the basis of their clinical diagnosis of psychosis, being the 'regular' screening arm in the T.TIP flow chart for the randomised controlled treatment study, not the 'incidental referrals' arm that was designed for potential participants who were suspected to have PTSD.'

b. As designed, ${ }^{7}$ only a random selection of participants with a TSQ score below six were interviewed. 


\begin{tabular}{|c|c|c|}
\hline & $\begin{array}{l}\text { Screened group } \\
\qquad(n=2608)\end{array}$ & $\begin{array}{l}\text { Interviewed group (MINI-Plus and CAPS) } \\
\qquad(n=455)\end{array}$ \\
\hline \multicolumn{3}{|l|}{ Age, years } \\
\hline Median & 43.0 & 41.0 \\
\hline Mean & 41.9 & 40.9 \\
\hline \multicolumn{3}{|l|}{ Gender, \% } \\
\hline Men & 61.8 & 60.0 \\
\hline Women & 38.2 & 40.0 \\
\hline \multicolumn{3}{|l|}{ Duration of illness (psychosis), years } \\
\hline Median & 13.0 & 13.0 \\
\hline Mean & 15.0 & 14.8 \\
\hline \multicolumn{3}{|l|}{ Primary chart diagnosis, $\%$} \\
\hline Schizophrenia & 54.3 & 49.1 \\
\hline Schizoaffective disorder & 10.9 & 11.8 \\
\hline Delusional disorder, psychotic disorder NOS, brief psychotic disorder & 21.3 & 26.7 \\
\hline Bipolar disorder with psychotic features & 6.9 & 4.6 \\
\hline Depressive disorder with psychotic features & 3.8 & 5.8 \\
\hline Other ${ }^{a, b}$ & 2.8 & 2.0 \\
\hline Legal status, involuntary treatment: \% & 6.4 & 3.7 \\
\hline \multicolumn{3}{|l|}{ Housing, \% } \\
\hline Independent & 79.5 & 83.1 \\
\hline Sheltered & 12.9 & 10.0 \\
\hline Hospital & 4.2 & 3.9 \\
\hline Parents & 3.1 & 2.1 \\
\hline Others & 0.3 & 0.9 \\
\hline \multicolumn{3}{|c|}{$\begin{array}{l}\text { NOS, not otherwise specified. } \\
\text { a. For Primary chart diagnosis in the screened sample the } 2.8 \% \text { 'Other' category includes } 0.1 \% \text { PTSD. In } 0.4 \% \text { of the screened sample, PTSD was classified in the charts as the } \\
\text { secondary diagnosis (data not shown) and in } 0.0 \% \text { as the tertiary diagnosis (data not shown). Added together, a classification of PTSD in the chart diagnosis was present in } 0.5 \% \\
\text { (13 people) of the total screened sample. } \\
\text { b. If a psychotic disorder was not the Primary chart diagnosis, then a psychotic disorder was recorded as the second or third diagnosis (data not shown). }\end{array}$} \\
\hline
\end{tabular}

with the MINI-Plus and CAPS $(n=455)$ (Fig. 1). Table 1 shows that our samples are representative of the target population regarding age, gender distribution, diagnosis and duration of illness.

\section{Trauma exposure in patients with psychotic disorders}

Table 2 presents the results on trauma exposure, i.e. the prevalence of exposure to any trauma and exposure per trauma category, in the total sample and in women and men separately. The prevalence of trauma exposure was $78.2 \%$, and $55.9 \%$ of the patients reported exposure to three or more traumatic incidents. The mean number of trauma categories (such as 'physical abuse') that patients selected was 2.2. These results indicate that this population is a multiple traumatised population. The logistic regression analysis enter method, with gender as a predictor, demonstrated significant gender differences. In this sample, women were more likely than men to report any kind of trauma exposure (odds ratio $(\mathrm{OR})=1.76$ ), sexual abuse $(\mathrm{OR}=3.58)$, physical abuse $(\mathrm{OR}=1.47)$ and emotional abuse $\mathrm{OR}=1.58)$. Incidents of accident/disaster/war were reported less frequently by women $(\mathrm{OR}=0.83)$ than by men.

\section{Predictive validity of the TSQ}

Predictor variables by logistic regression

The logistic regression model included seven possible predictors. The first predictor was 'gender' because, in the Netherlands, the odds ratio for women compared with men to develop PTSD after trauma was found to be 2.00. ${ }^{21}$ The predictors were 'sexual abuse', 'physical abuse,' 'emotional or psychological abuse', 'severe neglect' and 'accident/disaster/war' because these five trauma categories were highly prevalent in our sample. Most of these variables are known for their prevalence in people with psychotic disorders. $^{22-24}$ Finally, the seventh predictor to be entered was the TSQ total score. The trauma category 'traumatic psychosis' was excluded from the elimination procedure; the predictive quality of this variable was per definition limited because all participants had experienced psychosis by inclusion. Table 3 shows that four of the predictor variables made a unique significant contribution to the goodness of fit of the model: sexual abuse, physical abuse, severe neglect (negative association) and TSQ total score.

The goodness of fit of the predictor model as a whole (Omnibus test of model coefficients) was significant, $\chi^{2}(7, n=455)=137.25$, $P<0.001$. The model correctly classified $73.3 \%$ of the cases as opposed to $64.4 \%$ predicted correctly from the null model. The pseudo $R^{2}$ statistics of Cox and Snell $R^{2}(0.26)$ and the Nagelkerke $R^{2}$ (0.35) suggest that $26.0-35.8 \%$ of the variance in PTSD is explained by the variables in the model. The Hosmer-Lemeshow goodness-of-fit test yielded significant support for the model, $\chi^{2}(8, n=455)=7.92, P=0.441$ (note that, in this test, high probability values of $P>0.05$ indicate support for the model).

The selected predictor variables (sexual abuse, physical abuse, severe neglect and TSQ total score) estimated the odds of having a PTSD, or not, for each individual in the screened sample. Probabilities $\geqslant 0.5$ were imputed as 'PTSD'; probabilities $<0.5$ were imputed as 'no PTSD'. We considered the pooled results of this multiple imputation procedure to be an estimate of the prevalence of PTSD in this sample of patients with psychotic disorders.

\section{Estimated PTSD prevalence}

The procedure described above yielded an estimated PTSD prevalence in the total screened sample $(n=2608)$ of $16.0 \%$ (i.e. 416 individuals, $95 \%$ CI $14.6-17.4 \%$ ). Significantly more women $(49.3 \%)$ than men $(50.7 \%)$ have estimated PTSD in comparison 


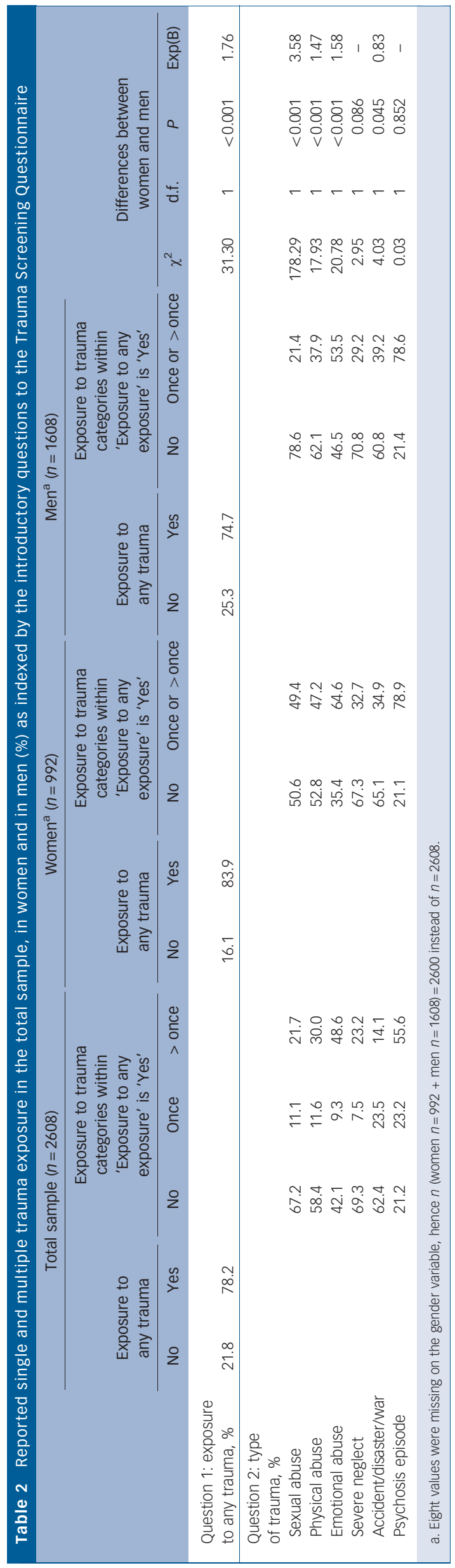

with the characteristics of the screening sample (Table 1, women $38.2 \%$, men $61.8 \%,\left(\chi^{2}(1, n=2608)=14.446, P<0.001\right)$. The significant gender difference in estimated PTSD prevalence closely resembles the gender difference in actual PTSD prevalence found in the CAPS interviewed group ( $n=455)$ : more women $(50 \%)$ and less men $(50 \%)$ than expected (Table 1, men $60.0 \%$, women $40.0 \%$ ) reached a positive diagnostic PTSD status $\left(\chi^{2}(1, n=455)=6.578\right.$, $P=0.010)$.

\section{Clinically recorded PTSD prevalence}

The mental health organisations recorded DSM-IV-PTSD classifications in $0.5 \%$ ( 13 patients) of the clinical charts of all 2608 participants, either as the primary, secondary or tertiary Axis 1 diagnosis. In 12 of these 13 patients (92\%), the PTSD diagnosis in the clinical charts corresponded with the diagnosis as assessed with the CAPS in the present study.

\section{Psychometric quality of the TSQ as predictor of PTSD}

Comparing TSQ total scores with PTSD status yields the ROC curve presented in Fig. 2. The area under the curve (AUC $=0.85,95 \%$ CI $0.83-0.87, P \leqslant 0.001$ ) was significant for the TSQ total score. Table 4 presents the psychometric properties, i.e. the PTSD classification accuracy, for each of the 10 possible TSQ cut-off scores. A cut-off score of 6 achieves the best mean of the sum of sensitivity (78.8\%) and specificity (75.6\%) and yields $44.5 \%$ correct positive and $93.6 \%$ correct negative predictions.

\section{Discussion}

\section{Trauma exposure in patients with psychotic disorders}

The reported prevalence of trauma exposure in our study population $(78.2 \%)$ proved to be similar to the estimated prevalence within the general population in the Netherlands (i.e. an $80.7 \%$ lifetime prevalence of any potential trauma). ${ }^{21}$ In the general population, women do not show a higher overall rate of trauma exposure than men. ${ }^{25}$ This is in contrast to the population of patients with psychotic disorders. Women reported a higher general rate of trauma exposure than men (Table 2) and more often reported (multiple) traumas that are known ${ }^{25}$ for their high risk of developing PTSD, particularly sexual and physical abuse.

\section{The experience of a traumatic psychosis}

In the Method section, we explained the relevance of adding 'an episode of psychosis' to the screening questions. Of the $78.2 \%$ participants who indicated having experienced 'any trauma', $78.8 \%$ reported that one $(23.2 \%)$ or more than one $(55.6 \%)$ episode of psychosis had been experienced as really frightening, life-threatening, overwhelming or shocking. From the available data we cannot infer the unique contribution of traumatic psychotic experiences to the PTSD symptoms reported in the TSQ screening. However, experiencing psychosis and its consequences is clearly very unsettling and, consequently, may contribute to the development of PTSD. For this specific patient population, extremely distressing experiences during their psychotic episodes, especially when they are experienced as lifethreatening, may be included as a traumatic event as indicated by the DSM-IV-PTSD-criterion A. 


\begin{tabular}{|c|c|c|c|c|c|c|}
\hline Predictors & $B$ & S.E. & Wald & d.f. & $P$ & $\operatorname{Exp}(\mathrm{B}), \mathrm{OR}(95 \% \mathrm{Cl})$ \\
\hline \multicolumn{7}{|l|}{ Predictor modela } \\
\hline Sexual abuse & & & 12.228 & 2 & 0.002 & \\
\hline Once & 0.688 & 0.349 & 3.889 & 1 & 0.049 & $1.990(1.004-3.942)$ \\
\hline More than once & 0.910 & 0.270 & 11.335 & 1 & 0.001 & $2.485(1.463-4.220)$ \\
\hline Physical abuse & & & 14.998 & 2 & 0.001 & \\
\hline Once & 0.678 & 0.386 & 3.090 & 1 & 0.079 & $1.971(0.925-4.198)$ \\
\hline More than once & 1.076 & 0.279 & 14.903 & 1 & $<0.001$ & $2.933(1.698-5.064)$ \\
\hline Severe neglect & & & 6.745 & 2 & 0.034 & \\
\hline Once & -0.185 & 0.443 & 0.174 & 1 & 0.676 & $0.831(0.349-1.981)$ \\
\hline More than once & -0.710 & 0.274 & 6.742 & 1 & 0.009 & $0.491(0.288-0.840)$ \\
\hline TSQ - total score & 0.431 & 0.060 & 51.539 & 1 & $<0.001$ & $1.538(1.367-1.730)$ \\
\hline \multicolumn{7}{|l|}{ Not in the predictor model } \\
\hline Emotional or psychological abuse & & & 2.617 & 2 & 0.454 & \\
\hline Once & 0.369 & 0.506 & 0.531 & 1 & 0.466 & $1.446(0.536-3.897)$ \\
\hline More than once & 0.153 & 0.384 & 0.157 & 1 & 0.692 & $1.165(0.548-2.475)$ \\
\hline Accident/disaster/war & & & 1.188 & 2 & 0.756 & \\
\hline Once & -0.307 & 0.291 & 1.113 & 1 & 0.291 & $0.736(0.416-1.301)$ \\
\hline More than once & -0.174 & 0.308 & 0.321 & 1 & 0.571 & $0.840(0.459-1.536)$ \\
\hline Gender & 0.260 & 0.247 & 1.106 & 1 & 0.293 & $1.297(0.799-2.103)$ \\
\hline
\end{tabular}

\section{Estimated PTSD prevalence in the population of patients with psychotic disorders}

In the present study, the estimated prevalence of comorbid PTSD in patients with psychotic disorders (16\%) is at the higher end, but well within the prevalence range of $12.4 \%(95 \%$ CI $4.0-20.8 \%$ ) that was found in a meta-analysis. ${ }^{1}$ These results corroborate previous findings indicating that PTSD is more common in individuals with a psychotic disorder than in the general population, for example a $3.3 \%$ PTSD prevalence in the Dutch general population. ${ }^{21}$ One explanation for this could be that patients with psychotic disorders are more vulnerable to developing PTSD because of deficits in information processing. ${ }^{26}$ Another hypothesis is that patients with psychotic disorders more often encounter multiple childhood traumas, ${ }^{22,23,27}$ which may form particular psychological and neurodevelopmental ${ }^{28}$ pathways that lead from childhood adversity to psychosis.

As in the general population, ${ }^{19}$ female patients with psychotic disorders experience PTSD significantly more often than men. Note, however, that our logistic regression analysis (Table 3) indicates that it is not the gender of the person subjected to trauma exposure that best predicts PTSD. Rather, PTSD is best predicted by the type and frequency of the specific events that a person has been exposed to, particularly (repeated) sexual or physical abuse, and by the TSQ total score reflecting the severity of screened PTSD symptoms. Future research should provide more insight into gender differences and effects in pathways from trauma-exposure characteristics to PTSD in patients with psychotic disorders.

\section{Clinical documentation of PTSD in patients with psychotic disorders}

Surprisingly, the estimated prevalence of 16\% PTSD (417 individuals) in our total sample of patients with psychotic disorders is 32 times higher than the $0.5 \%$ DSM-IV-TR PTSD classifications (13 individuals) recorded by the mental health organisations (footnote to Table 1). Missing $15.5 \%$ of $16 \%$ of people with PTSD indicates an estimated under-report in clinical practice of no less than $96.9 \%$ of all those with PTSD. This finding is in line with a previous study ${ }^{2}$ and should raise serious concerns. Despite the extensive reports on the various causal pathways, and negatively mutually reinforcing interactions between trauma, (re)victimisation, PTSD and psychosis, ${ }^{22,23,29,30}$ this literature seems to be neglected by clinicians in the field of psychosis. An important reason for the under-reporting of PTSD might be the hierarchical system of diagnosis. Once a psychotic disorder (for example schizophrenia) has been diagnosed, other diagnoses are often not considered. ${ }^{31}$ Another explanation for the underdiagnosis in clinical practice might be that, unlike patients ${ }^{32}$ and some groundbreaking professionals (see for example Cusack et $a l^{31}$ and Read et $\mathrm{al}^{33}$ ), most mental health professionals are hesitant to discuss traumatic events with patients with psychosis. Many professionals fear that they may do harm, ${ }^{3,34}$ even though there is no evidence that adverse events might occur as a consequence of discussing trauma. ${ }^{35-37}$ Such an attitude is likely to prevent adequate

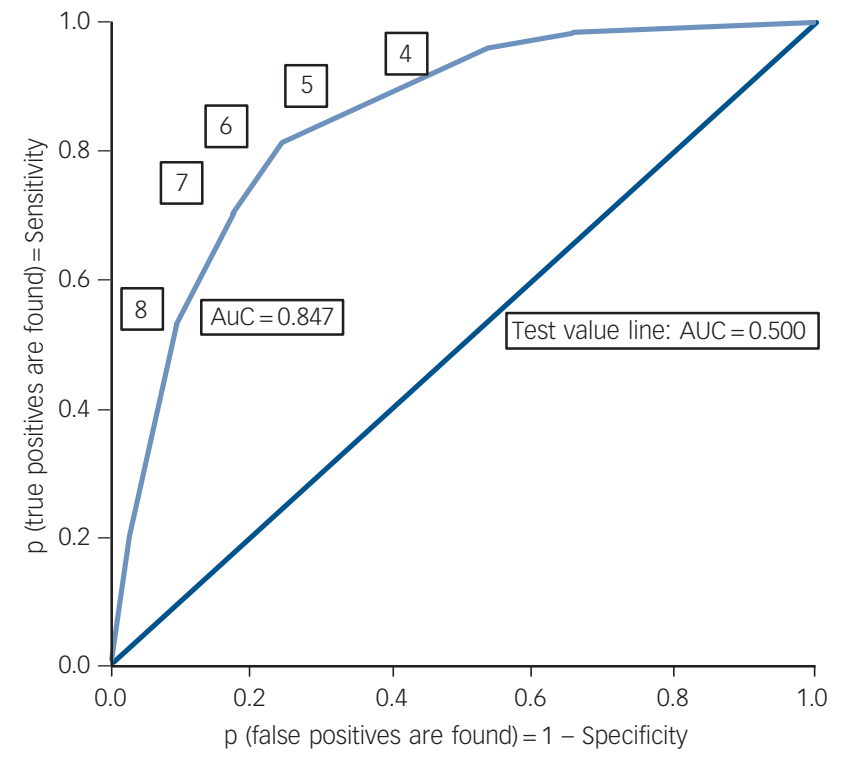

Fig. 2 Receiver operating characteristic (ROC) curve for cut-off scores and for area under the curve (AUC) of the Trauma Screening Questionnaire (TSQ) total score to predict post-traumatic stress disorder (PTSD) or no PTSD in patients with psychosis. 
Table 4 Classification accuracy of the Trauma Screening Questionnaire (TSQ) in predicting post-traumatic stress disorder (PTSD) V. no PTSD in adult with psychotic disorders

\begin{tabular}{|c|c|c|c|c|c|c|c|c|}
\hline $\begin{array}{c}\text { TSQ } \\
\text { cut-off score }\end{array}$ & $\begin{array}{l}\text { Sensitivity, } \\
\%\end{array}$ & $\begin{array}{l}\text { Specificity, } \\
\%\end{array}$ & $\begin{array}{c}\text { Positive predictive } \\
\text { value, } \%\end{array}$ & $\begin{array}{c}\text { Negative predictive } \\
\text { value, \% }\end{array}$ & $\begin{array}{l}\text { Positive likelihood } \\
\text { ratio, \% }\end{array}$ & $\begin{array}{c}\text { Accuracy, } \\
\%\end{array}$ & $\begin{array}{l}\text { Area under } \\
\text { the curve }\end{array}$ & $P$ \\
\hline$\geqslant 0$ & 100 & 0 & 20.0 & - & 1.0 & 20.0 & 0.85 & $<0.001$ \\
\hline$\geqslant 1$ & 98.0 & 23.9 & 24.3 & 97.9 & 1.3 & 38.7 & 0.85 & $<0.001$ \\
\hline$\geqslant 2$ & 96.5 & 35.4 & 27.1 & 97.6 & 1.5 & 47.6 & 0.85 & $<0.001$ \\
\hline$\geqslant 3$ & 94.9 & 46.8 & 30.8 & 97.4 & 1.8 & 56.4 & 0.85 & $<0.001$ \\
\hline$\geqslant 4$ & 91.3 & 55.8 & 34.0 & 96.2 & 2.1 & 62.8 & 0.85 & $<0.001$ \\
\hline$\geqslant 5$ & 85.3 & 66.4 & 38.7 & 94.8 & 2.5 & 70.2 & 0.85 & $<0.001$ \\
\hline$\geqslant 6^{a}$ & 78.8 & 75.6 & 44.5 & 93.6 & 3.2 & 76.3 & 0.85 & $<0.001$ \\
\hline$\geqslant 7$ & 64.6 & 83.0 & 48.6 & 90.4 & 3.8 & 79.3 & 0.85 & $<0.001$ \\
\hline$\geqslant 8$ & 48.8 & 90.1 & 55.5 & 87.8 & 5.0 & 82.0 & 0.85 & $<0.001$ \\
\hline$\geqslant 9$ & 31.3 & 94.4 & 58.3 & 84.6 & 5.6 & 81.8 & 0.85 & $<0.001$ \\
\hline 10 & 15.3 & 97.9 & 64.0 & 82.2 & 7.1 & 81.4 & 0.85 & $<0.001$ \\
\hline
\end{tabular}

diagnosis and treatment of comorbid PTSD, thereby needlessly prolonging the adverse consequences of untreated PTSD.

\section{The TSQ as a valid predictor of PTSD in patients with psychotic disorders}

In this group of patients, the TSQ demonstrated reasonably good psychometric properties. This instrument performs as well in the multi-trauma population in the present study as it does in population samples selected on the basis of sharing the same (single) trauma. ${ }^{5,10-12}$ Accordingly, incorporating the TSQ into a PTSD detection procedure will probably save time and expenditure. Interviewing every patient with a psychotic disorder for PTSD is not a viable option for most mental health services; however, screening for PTSD using the validated TSQ takes only a few minutes and is therefore an efficient option. More specifically, only $35 \%$ of the 2608 patients scored on or above the TSQ cut-off score for possible PTSD, reducing the need for CAPS interviewing by $65 \%$. The TSQ's high negative predictive value $(93.6 \%)$ indicates that very few patients with PTSD were missed because of false-negative exclusion. Subsequently, nearly half of the $35 \%$ of patients with a high TSQ score were diagnosed with PTSD based on the CAPS, indicating that on average just over two CAPS interviews were needed to detect PTSD in one patient. Although the cut-off score we found is in line with most previous studies, ${ }^{5,10,11}$ further research into the performance of the TSQ in these patients is warranted.

From a clinical point of view, it is important to note that the caregivers of the participating patients did not report any 'serious adverse events' (i.e. suicide, suicide attempt, self-mutilation with need for medical care, admissions to hospital, aggression with restraint) as a consequence of filling in the screening questionnaire. In fact, many patients reported the opposite and expressed gratitude that their traumas and related symptoms had been validated in this study's assessment procedure.

\section{Strengths and limitations}

To the best of our knowledge, this is the first study to examine screening for PTSD in patients using mental health services who are severely mentally ill with a diagnosis of psychotic disorders. The study included a large sample from a long-term care population with chronic psychotic disorders. The fact that the TSQ was tested against PTSD diagnostic status using the CAPS as the gold standard contributed considerably to the credibility of the TSQ's validity. Nevertheless, it could be argued that the $16 \%$ prevalence of PTSD reported in this study is only an estimate. Indeed, it was not a feasible option to interview all screened participants with the CAPS. However, considering the large sample sizes of both the screened group $(n=2608)$ and the interviewed group $(n=455)$, the narrow confidence interval of $14.6-17.4 \%$ and the fact that the prevalence rate is comparable with that reported in a meta-analysis, ${ }^{1}$ the PTSD estimate of $16 \%$ seems relatively robust and credible.

\section{Implications}

The TSQ is a valid PTSD screening tool for use in patients with psychotic disorders. Further, it is very short, rarely misses PTSD and can be used in multiple traumatised, complex patient groups with a wide variety of trauma types and frequencies that patients may or may not have experienced. The use of a screening tool such as the TSQ may be an important aid in reducing the high rate of underdiagnosis of PTSD in large cohorts of patients. Hopefully, the detection of PTSD will boost the application of PTSD treatments, such as prolonged exposure and eye movement desensitisation and reprocessing, ${ }^{38-42}$ which has been proven safe in patients with psychotic disorder.

Paul A. J. M. de Bont, MSC, Community Mental Health Service GGZ Oost Brabant Land van Cuijk en Noord Limburg, Boxmeer and Radboud University Nijmegen, Behavioural Science Institute, NijCare, The Netherlands; David P. G. van den Berg MSc, Parnassia Psychiatric Institute, Den Haag, The Netherlands; Berber M. van der Vleugel, MSC, Community Mental Health Service GGZ Noord-Holland Noord, Alkmaar, The Netherlands; Carlijn de Roos, MSC, MHO Rivierduinen, Leiden, The Netherlands; Ad de Jongh, PhD, Department of Behavioral Sciences, Academic Centre for Dentistry Amsterdam (ACTA), University of Amsterdam and VU University Amsterdam, Amsterdam, The Netherlands and School of Health Sciences, Salford University, Manchester, UK; Mark van der Gaag, PhD, VU University Amsterdam and EMGO Institute for Health and Care Research, Department of Clinical Psychology, Amsterdam and Parnassia Psychiatric Institute, Den Haag, The Netherlands; Agnes van Minnen, PhD, Radboud University Nijmegen, Behavioural Science Institute, NijCare and MHO 'Pro Persona', Centre for Anxiety Disorders Netherlands, Nijmegen, The Netherlands

Correspondence: Paul A. J. M. de Bont, Postbox 103, 5830 AC Boxmeer, The Netherlands. Email address: paj.de.bont@gzoostbrabant.n

First received 18 Mar 2014, final revision 19 Nov 2014, accepted 15 Jan 2015

\section{Funding}

The T.TIP trial, including the TSQ study, is supported by the Dutch Support Foundation 'Stichting tot Steun VCVGZ' (awarded to the principal investigator M.v.d.G.). The Dutch Support Foundation 'Stichting tot Steun VCVGZ' played no further role in any of the aspects or phases of this study.

\section{Acknowledgements}

The authors thank all the T.TIP participants, research assistants and the many mental health workers who contributed to this study. Special thanks go to Ms Marion Bruns and Ms Daniëlle Tilburgs for organising and preparing the data collection. We also thank Ms Daniëlle Tilburgs for organising and preparing the data collection. We also thank
the Royal College of Psychiatrists for their permission to republish the original Trauma screening Questionnaire with the additional questions that were relevant to this study. 
Appendix

\section{Introductory questions about trauma exposure and the Trauma Screening Questionnaire}

Question 1

Have you ever experienced events in your life, or have you ever witnessed events that were really frightening, life-threatening, overwhelming or shocking?

(Tick the appropriate answer)
No
(Thank you for your participation, you may hand in your questionnaire)
Yes
(Please continue to question 2)

Question 2

What kind of shocking experience(s) have you experienced?

(Tick what is applicable to you; you may tick more than one answer)

\begin{tabular}{|l|l|l|}
\hline & $\begin{array}{c}\text { Yes, one } \\
\text { traumatic } \\
\text { experience }\end{array}$ & $\begin{array}{c}\text { Yes, more than } \\
\text { one traumatic } \\
\text { experience }\end{array}$ \\
\hline Sexual activities against your will & & \\
\hline Physical abuse & & \\
\hline Emotional or psychological abuse & & \\
\hline Severe neglect & & \\
\hline Accident/disaster/war & & \\
\hline An episode of psychosis & & \\
\hline
\end{tabular}

Question 3

\section{Trauma Screening Questionnaire}

Your own reactions now to the traumatic event

Please consider the following reactions which sometimes occur after a traumatic event. This questionnaire is concerned with your personal reactions to the traumatic event which happened to you. Please indicate (Yes/No) whether or not you have experienced any of the following at least twice in the past week.

\begin{tabular}{|c|c|c|c|}
\hline & & \multicolumn{2}{|c|}{ At least twice in the past week? } \\
\hline & & YES & NO \\
\hline 1 & Upsetting thoughts or memories about the event that have come into your mind against your will & & \\
\hline 2 & Upsetting dreams about the event & & \\
\hline 3 & Acting or feeling as though the event were happening again & & \\
\hline 4 & Feeling upset by reminders of the event & & \\
\hline 5 & $\begin{array}{l}\text { Bodily reactions (such as fast heartbeat, stomach churning, sweatiness, dizziness) when reminded of the } \\
\text { event }\end{array}$ & & \\
\hline 6 & Difficulty falling or staying asleep & & \\
\hline 7 & Irritability or outbursts of anger & & \\
\hline 8 & Difficulty concentrating & & \\
\hline 9 & Heightened awareness of potential dangers to yourself and others & & \\
\hline 10 & Being jumpy or being startled at something unexpected & & \\
\hline
\end{tabular}

(C) 2002 The Royal College of Psychiatrists. Brewin CR, Rose S, Andrews B, Green J, Tata P, McEvedy C, et al. Brief screening instrument for post-traumatic stress disorder. British Journal of Psychiatry 2002; 181: 158-62. Written permission must be obtained from the Royal College of Psychiatrists for copying and distribution to others or for republication (in print, online or by any other medium).

\section{References}

1 Achim AM, Maziade M, Raymond E, Olivier D, Merette C, Roy M-A. How prevalent are anxiety disorders in schizophrenia? A meta-analysis and critical review on a significant association. Schizophr Bull 2011; 37: 811-21.

2 Lommen MJJ, Restifo K. Trauma and posttraumatic stress disorder (PTSD) in patients with schizophrenia or schizoaffective disorder. Community Ment Health J 2009; 45: 485-96.

3 Becker $\mathrm{CB}$, Zayfert C, Anderson E. A survey of psychologists' attitudes towards and utilization of exposure therapy for PTSD. Behav Res Ther 2004; 42: 277-92.
4 Ronconi JM, Shiner B, Watts, BV. Inclusion and exclusion criteria in randomized controlled trials of psychotherapy for PTSD. J Psychiatric Pract 2014; 20: 25-37.

5 Brewin CR, Rose S, Andrews B, Green J, Tata P, McEvedy C, et al. Brief screening instrument for post-traumatic stress disorder. Br J Psychiatry 2002; 181: $158-62$.

6 American Psychiatric Association. Diagnostic and Statistical Manual of Mental Disorders (4th edn, revised) (DSM-IV-TR). APA, 2000.

7 Blake DD, Weathers FW, Nagy LM, Kaloupek DG, Gusman FD, Charney DS, et al. The development of a Clinician-Administered PTSD Scale. J Trauma Stress 1995; 8: 75-90. 
8 de Bont PAJM, van den Berg DPG, van der Vleugel BM, de Roos C, Mulder CL, Becker ES, et al. A multi-site single blind clinical study to compare the effects of prolonged exposure, eye movement desensitization and reprocessing and waiting list on patients with a current diagnosis of psychosis and comorbid post traumatic stress disorder: study protocol for the randomized controlled trial Treating Trauma in Psychosis. Trials 2013; 14: 151

9 Foa EB, Riggs DS, Dancu CV, Rothbaum BO. Reliability and validity of a brie instrument for assessing post-traumatic stress disorder. J Trauma Stress 1993; 6: 459-73.

10 Brewin CR, Fuchkan N, Huntley Z, Scragg P. Diagnostic accuracy of the Trauma Screening Questionnaire after the 2005 London bombings. J Trauma Stress 2010; 23: 393-8.

11 Walters JTR, Bisson Jl, Shepherd JP. Predicting post-traumatic stress disorder: validation of the Trauma Screening Questionnaire in victims of assault. Psychol Med 2007; 37: 143-50.

12 Dekkers AMM, Olff M, Naring GWB. Identifying persons at risk for PTSD after trauma with TSQ in The Netherlands. Community Ment Health J 2010; 46: 20-5.

13 Lecrubier $\mathrm{Y}$, Sheehan DV, Weiller E, Amorim P, Bonora I, Harnett Sheehan $\mathrm{K}$ et al. The Mini International Neuropsychiatric Interview (MINI). A short diagnostic structured interview: reliability and validity according to the CIDI. Eur Psychiatry 1997; 12: 224-31.

14 Sheehan DV, Lecrubier Y, Harnett Sheehan K, Janavs J, Weiller E, Keskiner A et al. The validity of the Mini International Neuropsychiatric Interview (MINI) according to the SCID-P and its reliability. Eur Psychiatry 1997; 12: 232-41.

15 Van Vliet I, De Beurs E. Het Mini International Neuropsychiatric Interview (MINI). Een kort gestructureerd diagnostisch psychiatrisch interview voor DSM-IV- en ICD-10-stoornissen [The Mini-International Neuropsychiatric Interview. A brief structured diagnostic psychiatric interview for DSM-IV and ICD-10 psychiatric disorders.] Tijdschrift voor Psychiatrie 2007; 49: 393-7.

16 International Society for Traumatic Stress Studies. Clinician-Administered PTSD Scale (CAPS). ISTSS, 2013 (http://www.istss.org/ ClinicianAdministeredPTSDScaleCAPS.htm).

17 Weathers FW, Keane TM, Davidson JR. Clinician-administered PTSD scale: a review of the first ten years of research. Depress Anxiety 2001; 13: 132-56.

18 Weathers FW, \& Keane TM (2007). The Criterion A problem revisited: controversies and challenges in defining and measuring psychological trauma. J Trauma Stress 2007; 20: 107-21.

19 Shaw K, McFarlane AC, Bookless C, Air T. The aetiology of postpsychotic posttraumatic stress disorder following a psychotic episode. J Trauma Stress 2002; 15: 39-47.

20 Weathers FW, Ruscio AM, Keane TM. Psychometric properties of nine scoring rules for the Clinician-Administered Posttraumatic Stress Disorder Scale. Psychol Assessment 1999; 11: 124-33.

21 de Vries G-J, Olff M. The lifetime prevalence of traumatic events and posttraumatic stress disorder in The Netherlands. J Trauma Stress 2009; 22 259-67.

22 Varese $F$, Smeets $F$, Drukker $M$, Lieverse $R$, Lataster $T$, Viechtbauer $W$, et al. Childhood adversities increase the risk of psychosis: a meta-analysis of patient-control, prospective- and cross-sectional cohort studies. Schizophr Bull 2012; 29: 661-71.

23 Read J, Os J, Morrison AP, Ross CA. Childhood trauma, psychosis and schizophrenia: a literature review with theoretical and clinical implications. Acta Psychiatr Scand 2005; 112: 330-50.
24 Sautter FJ, Brailey K, Uddo MM, Hamilton MF, Beard MG, Borges AH. PTSD and comorbid psychotic disorder: comparison with veterans diagnosed with PTSD or psychotic disorder. J Trauma Stress 1999; 12: 73-88.

25 Olff M, Langeland W, Draijer N, Gersons BPR. Gender differences in posttraumatic stress disorder. Psychol Bull 2007; 133: 183-204.

26 Steel C, Fowler D, Holmes EA. Trauma-related intrusions and psychosis: an information-processing account. Behav Cogn Psychother 2005; 33 : 139-52.

27 Galletly C, Van Hooff M, McFarlane A. Psychotic symptoms in young adults exposed to childhood trauma - a 20 year follow-up study. Schizophr Res 2011; 127: 76-82.

28 Read J, Fosse R, Moskowitz A, Perry B. The traumagenic neurodevelopmental model of psychosis revisited. Neuropsychiatry 2014; 4: 65-79.

29 Morrison AP, Frame L, Larkin W. Relationships between trauma and psychosis: a review and integration. Br J Clin Psychol 2003; 42: 331-53.

30 Classen CC, Palesh OG, Aggarwal R. Sexual revictimization: a review of the empirical literature. Trauma Violence Abuse 2005; 6: 103-29.

31 Cusack KJ, Wells CB, Grubaugh A L, Hiers TG, Frueh BC. An update on the South Carolina trauma initiative. Psychiatr Serv 2007; 58: 708-10.

32 Lothian J, Read J. Asking about abuse during mental health assessments: clients' views and experiences. New Zeal J Psychol 2002; 31: 98-103.

33 Read J, Hammersley P, Rudegeair T. Why, when and how to ask about childhood abuse. Adv Psychiatr Treat 2007; 13: 101-10.

34 van Minnen A, Hendriks L, \& Olff M. When do trauma experts choose exposure therapy for PTSD patients? A controlled study of therapist and patient factors. Behav Res Ther 2010; 48: 312-20.

35 Grubaugh AL, Tuerk PW, Egede LE, Frueh BC. Perceptions of PTSD research participation among patients with severe mental illness. Psychiatry Res 2012; 200: 1071-3.

36 Brown VM, Strauss JL, LaBar KS, Gold AL, McCarthy G, Morey RA. Acute effects of trauma-focused research procedures on participant safety and distress. Psychiatry Res 2014; 215: 154-8.

37 van Minnen A, Harned MS, Zoellner L, Mills K. Examining potential contraindications for prolonged exposure therapy for PTSD. Eur J Psychotraumatol 2012; July 25 (epub ahead of print).

38 Frueh CB, Grubaugh AL, Cusack KJ, Kimble MO, Elhai JD, Knapp RG. Exposure-based cognitive-behavioral treatment of PTSD in adults with schizophrenia or schizoaffective disorder: pilot study. J Anxiety Disord 2009; 23: $665-75$.

39 Mueser KT, Rosenberg SD, Xie H, Jankowski MK, Bolton EE, Lu W, et al. A randomized controlled trial of cognitive-behavioral treatment for posttraumatic stress disorder in severe mental illness. J Consult Clin Psychol 2008; 76: 259-71

40 van den Berg DPG, van der Gaag M. Treating trauma in psychosis with EMDR: a pilot study. J Behav Ther Exp Psychiatry 2012; 43: 664-71.

41 de Bont PA, van Minnen A, de Jongh A. Treating PTSD in patients with psychosis: a within-group controlled feasibility study examining the efficacy and safety of evidence-based PE and EMDR protocols. Behav Ther 2013; 44: 717-30

42 Van den Berg DP, De Bont PA, van der Vleugel BM, Roos $C$, de Jongh $A$ van Minnen $A$ et al. Prolonged exposure versus eye movement desensitization and reprocessing versus waiting list for posttraumatic stress disorder in patients with a psychotic disorder. A randomized controlled trial. JAMA Psychiatry, 21 January 2015 (doi: 10.1001/ jamapsychiatry.2014.2637). 